\title{
ВЛИЯНИЕ СМЕЩЕНИЯ СТАЦИОНАРНОГО ПОТЕНЦИАЛА АЛЮМИНИЕВОЙ ПОВЕРХНОСТИ НА СВОЙСТВА НИКЕЛЬ-ФОСФОРНЫХ ПОКРЫТИЙ
}

\author{
Рогова Инна Андреевна',
}

inna.aleksenk092@mail.ru

\author{
Ильин Александр Петрович', \\ ilyin@mail.ru
}

\author{
Налесник Олег Иванович', \\ nalesnik@tpu.ru
}

\author{
Матренин Сергей Вениаминович', \\ msv@mail.ru
' Национальный исследовательский Томский политехнический университет, Россия, 634050, г. Томск, пр. Ленина, 30.

\begin{abstract}
Актуальность исследования обусловлена необходимостью совершенствования уже известных методов нанесения химических покрытий. К настоящему времени, несмотря на многочисленные исследования и практическое применение, процесс химического никелирования остается неидеальным. Небольшая скорость осаждения никеля, высокий расход компонентов раствора, а также сложность проведения процесса нанесения не способствуют повсеместному применению химического никелирования. В то же время, получаемые химическим способом покрытия значительно отличаются от тех, что получают электрохимическим способом: обладают ценными свойствами. Предложенный способ интенсификации за счет совмещения процессов нанесения никеля химическим и электрохимическим способами способен увеличить скорость осаждения покрытий, а также позволяет влиять на их состав и механические свойства.

Цель: повышение скорости химического никелирования путем наложения при осаждении отрицательного электрического потенциала на алюминиевую подложку, с предварительно нанесенным тонким слоем химически осажденного никеля, для сокращения времени проведения процесса.

объект: никелевые покрытия на алюминиевой подложке из сплава марки Д16Т.

Методы: потенциодинамический, сканирующая электронная микроскопия, энергодисперсионный анализ, микротвердость, скретч-тест, профилометрия.

Результаты. Осуществлено совместное соосаждение химического и электрохимического никеля на алюминиевую подложку наложением на нее отрицательного электрического потенциала. Определено содержание фосфора в покрытиях в зависимости от смещения стационарного потенциала поверхности. Изучены физико-химические характеристики полученных покрытий: шероховатость, адгезия, твердость. Установлено, что небольшое смещение стационарного потенциала поверхности в катодную область позволяет получать никель-фосфорные покрытия, содержащие меньшее количество фосфора, но превосходящие по
\end{abstract} свойствам химическое покрытие.

\section{Ключевые слова:}

Потенциал смещения, скорость осаждения никеля, химическое никелирование, алюминий, шероховатость, твердость, адгезия.

\section{Введение}

Химические никель-фосфорные покрытия широко используются для защиты деталей машин и механизмов, так как обладают различными ценными свойствами, такими как: равномерность, однородность, коррозионная стойкость, твердость, износоустойчивость [1-4].

Химическое никелирование относится к процессам автокаталитического восстановления, протекающим по электрохимическому механизму, основанному на восстановлении ионов никеля гипофосфитом в водных растворах. Механизм реакций, протекающих при химическом никелировании, весьма сложен и до сих пор изучается и уточняется $[5,6]$.

Реакция восстановления никеля гипофосфитом имеет ступенчатый характер, следовательно, химическое никелирование алюминиевых сплавов характеризуется невысокой скоростью и быстрым затуханием процесса, а также низким коэффици- ентом использования солей никеля [7]. Соответственно, на скорость образования покрытия будет влиять множество факторов: концентрация основных компонентов, $\mathrm{pH}$ раствора, температура и время проведения процесса [8-10]. Следует отметить, что сам процесс химического никелирования занимает длительное время, при этом происходит расход химических реагентов, что, несомненно, является недостатком данного метода [11].

В данной работе рассмотрен способ повышения скорости химического никелирования за счет совмещения процессов нанесения никеля химическим и электрохимическим способами.

\section{Методики эксперимента}

Получение никель-фосфорного покрытия при совместном химическом и электрохимическом осаждении на алюминиевую поверхность осуществляли с использованием трехэлектродной системы 
и потенциостата П-5848. Электродом сравнения был хлорсеребряный, вспомогательным электродом - графитовый стержень, рабочим электродом - алюминиевая пластина. При проведении эксперимента в качестве электролита был выбран кислый сульфатный раствор химического никелирования [12].

Подготовка подложки к процессу включала: обезжиривание в четыреххлористом углероде и щелочном растворе, щелочное и кислотное травления, двойную цинкатную обработку с промежуточным кислотным травлением [13].

Вспомогательный и рабочий электроды, соединенные посредством солевого мостика с электродом сравнения, находящемся в растворе калия хлористого, погружали в предварительно разогретый до рабочей температуры $\left(80{ }^{\circ} \mathrm{C}\right)$ раствор электролита (250 мл).

После установления потенциала на алюминиевой пластине в течение трех минут проводили химическое никелирование (для формирования контактного никелевого слоя). На блоке задающих напряжений потенциостата устанавливали выбранную величину потенциала смещения, проводили соосаждение химического и электрохимического никеля в течение 30 минут. После нанесения покрытия пластину промывали в проточной воде, высушивали на воздухе и взвешивали. Определение средней скорости осаждения покрытия проводили весовым методом, согласно представленной ниже формуле:

$$
v_{N i}=\frac{\left(m_{2}-m_{1} k\right) \cdot 10000}{d \tau S},
$$

где $m_{1}$ - начальная масса детали, г; $m_{2}$ - конечная масса детали, г; $k$ - коэффициент стравливания равный 0,$9937 ; d-$ плотность никеля, г/ $\mathrm{cm}^{3} ; S-$ площадь одной детали, $\mathrm{cm}^{2} ; \tau$ - время проведения процесса, ч. Также проводили термическую обработку каждого образца при $350{ }^{\circ} \mathrm{C}$ в течение часа на воздухе.

Содержание фосфора в покрытии определяли с использованием энергодисперсионного анализа (Oxford INCA Energy 350). Твердость покрытий после отжига исследовали с помощью прибора Nano Indenter G200, в качестве индентора использовали алмазную пирамидку. Шероховатость покрытий исследовали с использованием трехмерного бесконтактного профилометра (Micro Measure 3D Station) с шагом измерения 0,1 мкм. Адгезионные свойства полученных образцов исследовали с помощью прибора Micro-Scratch Tester MST-S-AX0000. Диапазон нагрузки составлял от 0 до $15 \mathrm{H}$, скорость нагружения $15 \mathrm{H} /$ мин. Глубину проникновения определяли с помощью индукционного датчика. Звуки при растрескивании регистрировали акустическим датчиком.

\section{Результаты и их обсуждение}

Химическое никелирование алюминия происходит при постоянном стационарном потенциале, равном $-0,48$ В. При погружении алюминиевой пластины с цинковым подслоем в раствор химического никелирования происходит рост потенциала c $-0,90$ до $-0,48$ В за счет замещения цинкового подслоя (являющегося активатором) на никелевый. После установления стационарного потенциала, без осуществления каких-либо электрохимических воздействий, протекает процесс химического никелирования. При этом скорость процесса невелика, но при незначительном смещении стационарного потенциала в катодную область происходит увеличение скорости осаждения.

В работе была проведена серия экспериментов с целью установления влияния величины потенциала смещения на среднюю скорость осаждения никеля и содержание фосфора. Результаты экспериментов приведены в табл. 1.

Таблица 1. Скорость осаждения никеля и содержание фосфора в покрытии в зависимости от смещения потенциала

Table 1. Nickel deposition rate and phosphorus content in the coating in depend on the potential shift

\begin{tabular}{|c|c|c|}
\hline $\begin{array}{c}\text { Смешение } \\
\text { потенциала, B } \\
\text { Potential shift, V }\end{array}$ & $\begin{array}{c}\text { Скорость осаждения, } \\
\text { мкм/ч } \\
\text { Deposition rate, } \mu \mathrm{m} / \mathrm{h}\end{array}$ & $\begin{array}{c}\text { Содержание фосфора, } \\
\text { мac. \% }\end{array}$ \\
\hline 0,00 & 10 & 4,60 \\
\hline$-0,01$ & 14 & 4,05 \\
\hline$-0,03$ & 16 & 1,54 \\
\hline$-0,05$ & 18 & 3,68 \\
\hline$-0,08$ & 20 & 4,02 \\
\hline$-0,10$ & 22 & 1,78 \\
\hline$-0,15$ & 24 & 0 \\
\hline$-0,20$ & 30 & 0 \\
\hline$-0,25$ & 40 & 0 \\
\hline
\end{tabular}

Из полученных данных следует, что при сдвиге потенциала на сотые доли вольта в катодную область происходило увеличение скорости осаждения никеля. Поскольку при смещении потенциала происходило протекание электрического тока, то на химический процесс накладывался электрохимический, и доля химически осажденного никеля в составе осадка снижалась. Следовательно, должно наблюдаться изменение химического состава получаемого покрытия, а именно снижаться содержание фосфора. Это отражено в результатах (табл. 1), где при смещении потенциала на $-0,03$ В (при -0,51 В) происходило снижение содержания фосфора в покрытии. Дальнейшее смещение потенциала образца до $-0,08$ В вызывало рост содержания фосфора, что согласуется с содержанием электрохимически осажденного фосфора в покрытии.

При достижении смещения в $-0,10$ В и более количество фосфора в покрытии снижалось до уровня менее предела обнаружения фосфора (<0 мас. \%), что свидетельствовало о преимущественном содержании электрохимического никеля в сравнении с содержанием химического никеля в покрытии или высокой скоростью осаждения, при которой соосаждение фосфора просто невозможно. Соответственно, получение покрытия согласно механизму, представленному в [14], при совместном никелиро- 
вании с большим смещением стационарного потенциала в катодную область неосуществимо.

Катодное смещение потенциала подложки не только позволяет повышать скорость роста толщины никелевого покрытия (что позволяет сократить время получения толстослойных покрытий), но также способствует получению осадков с заданными составом и свойствами.

Морфология полученных образцов была исследована с использованием сканирующей электронной микроскопии. На рисунке приведена типичная микрофотография структуры поверхности химического никелевого покрытия: поверхность однородная, средний размер кристаллитов составляет 2 мкм. Ранее было показано, что при сдвиге стационарного потенциала наблюдается рост скорости осаждения никеля, поэтому изменяется и структура покрытия. Наиболее близкую структуру к химически осажденному покрытию имеет образец, полученный при катодном смещении в $-0,05$ В (рисунок, Б). Другие образцы, полученные при сдвиге до $-0,10$ В, отличаются от химически осажденного (рисунок, В). Покрытие, полученное при сдвиге потенциала на $-0,08$ В (рисунок, В), имеет неоднородную структуру поверхности за счет образования бугристых структур при автокаталитическом осаждении никеля в определенных точках поверхности. При сдвиге стационарного потенциала на $-0,10$ В (рисунок, Г) и более микроструктура поверхности разительно отличается от химически осажденного и близка к электрохимическому.

Полученные образцы отличались друг от друга по физико-химическим характеристикам, соответственно были проведены исследования зависимостей шероховатости, твердости и износостойкости покрытий от смещения стационарного потенциала поверхности.

В табл. 2 представлены результаты измерений величины шероховатости покрытия в зависимости от смещения стационарного потенциала поверхности, из которых следует, что с увеличением катодного смещения наблюдался как рост, так и снижение шероховатости.
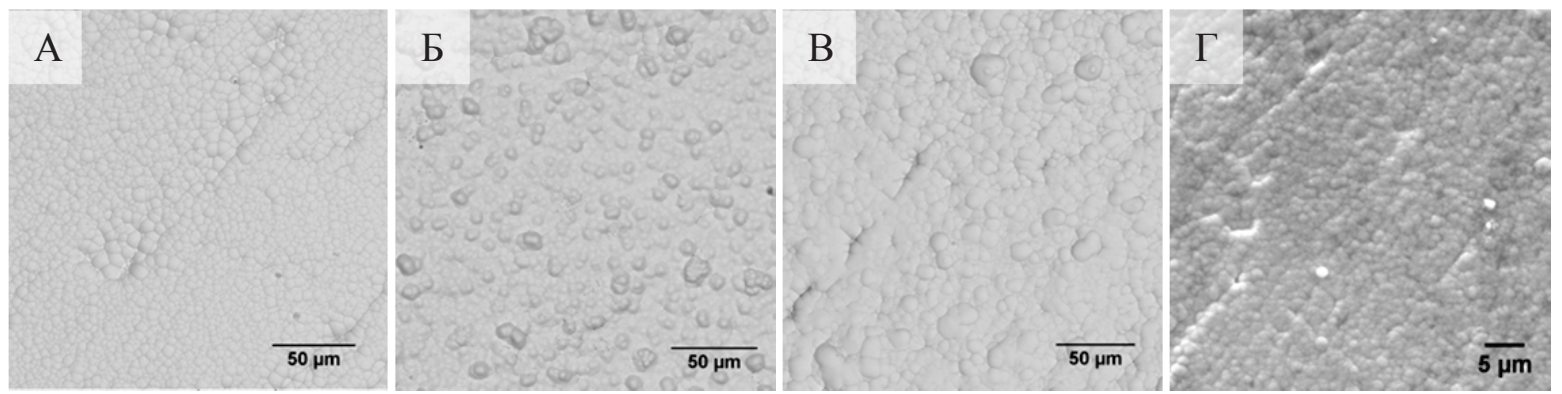

Рисунок. Микроструктура поверхности осажденных покрытий: (А) химическое никелирование; (Б) со сдвигом потенциала поверх ности на -0,05 B; (B) со сдвигом потенциала поверхности на -0,08 B; $(\Gamma)$ со сдвигом потенциала поверхности на -0,10 В

Figure. Surface microstructure of the deposited coatings: (A) electroless nickel; ( 5 ) with surface potential shift by $-0,05 \mathrm{~V}$; (B) with surface potential shift by $-0,08 \mathrm{~V} ;(\Gamma)$ with surface potential shift by $-0,10 \mathrm{~V}$ 
в которой атомы никеля и фосфора распределены равномерно [16]. Соответственно, при электрохимическом воздействии происходит неравномерное формирование никеля: осаждение происходит не по всей площади, а на отдельных кристаллах.

Согласно теоретическим данным, никелевые покрытия после получения необходимо термообрабатывать, так как нагрев способствует закрытию пор и протеканию процессов рекристаллизации в процессе отжига [17-20]. В табл. 3 представлены результаты зависимости микротвердости от смещения потенциала поверхности. Контрольными образцами были алюминиевая пластина и пластина, покрытая химическим никелированием.

Таблица 3. Микротвердость никелевого покрытия в зависилости от смещения потенииала поверхности

Table 3. Nickel coating microhardness in depend on the surface potential shift

\begin{tabular}{|r|c|}
\hline \multicolumn{1}{|c|}{$\begin{array}{l}\text { Образцы } \\
\text { Samples }\end{array}$} & $\begin{array}{c}\text { Микротвердость, МПа } \\
\text { Microhardness, MPa }\end{array}$ \\
\hline $\begin{array}{r}\text { алюминиевая пластина } \\
\text { aluminium plate }\end{array}$ & 1051 \\
\hline $\begin{array}{r}\text { химический никель } \mathrm{E}_{\text {кат }}=0,00 \mathrm{~B} \\
\text { electroless nickel } \mathrm{E}_{\text {cath }}=0,00 \mathrm{~V}\end{array}$ & 1097 \\
\hline $\begin{array}{r}\text { катодное смещение } \mathrm{E}_{\text {кат }}=0,01 \mathrm{~B} \\
\text { cathodic potential shift } \mathrm{E}_{\text {cath }}=0.01 \mathrm{~V}\end{array}$ & 587 \\
\hline $0,03 \mathrm{~B} / \mathrm{V}$ & 790 \\
\hline $0,05 \mathrm{~B} / \mathrm{V}$ & 1170 \\
\hline $0,08 \mathrm{~B} / \mathrm{V}$ & 1587 \\
\hline $0,10 \mathrm{~B} / \mathrm{V}$ & 1674 \\
\hline $0,15 \mathrm{~B} / \mathrm{V}$ & 2194 \\
\hline $0,20 \mathrm{~B} / \mathrm{V}$ & 4600 \\
\hline $0,25 \mathrm{~B} / \mathrm{V}$ & 6827 \\
\hline &
\end{tabular}

Образец покрытия, полученный при катодном смещении в $0,01 \mathrm{~B}$, обладал меньшей твердостью, чем контрольные образцы. Соответственно, небольшое электрохимическое воздействие на алюминиевую пластину ухудшает качество получаемых покрытий. При увеличении катодного сдвига твердость покрытий возрастала. Стоит отметить, что образец, полученный при смещении потенциала на 0,05 В, обладал твердостью выше контрольных образцов примерно на 100 МПа.

Дальнейшее смещение стационарного потенциала в катодную область, начиная с 0,08 В, способствовало получению покрытий с твердостью, которая превышала твердость контрольных образцов, что является положительным фактором для эксплуатационных характеристик покрытия.

Помимо шероховатости и микротвердости, одной из важных характеристик покрытий является адгезия. Соответственно, чем больше адгезия покрытия к подложке, тем дольше срок его службы.

В табл. 4 представлены результаты измерений адгезионных характеристик полученных никелевых покрытий.

Как видно из полученных данных, адгезионные характеристики росли с увеличением потенциала смещения поверхности до значения $-0,08$ В. После этого значения происходило снижение прочностных характеристик покрытий. При этом необходимо отметить, что незначительное электрохимическое воздействие в $0,01 \mathrm{~B}$ на поверхность не способствует увеличению прочностных характеристик.

Таблица 4. Адгезионные характеристики покрытий при различных потенииалах смешения

Table 4. Adhesive characteristics of the coating at various potential shifts

\begin{tabular}{|r|c|c|c|}
\hline \multirow{2}{*}{$\begin{array}{c}\text { Oбразцы } \\
\text { Samples }\end{array}$} & \multicolumn{3}{|c|}{ Усилие, H/Force, N } \\
\cline { 2 - 4 } & $\begin{array}{c}\text { Tрещины } \\
\text { Cracks }\end{array}$ & $\begin{array}{c}\text { Частичный } \\
\text { отрыв } \\
\text { Partial } \\
\text { coating loss }\end{array}$ & $\begin{array}{c}\text { Полный } \\
\text { отрыв } \\
\text { Overall } \\
\text { coating loss }\end{array}$ \\
\hline $\begin{array}{r}\text { химический никель } \mathrm{E}_{\text {кат }}=0,00 \mathrm{~B} \\
\text { electroless nickel } \mathrm{E}_{\text {саth }}=0,00 \mathrm{~V}\end{array}$ & 0,50 & 0,89 & 1,61 \\
\hline $\begin{array}{r}\text { катодное смещение } \mathrm{E}_{\text {кат }}=0,01 \mathrm{~B} \\
\text { cathodic shift } \mathrm{E}_{\text {саth }}=0,01 \mathrm{~V}\end{array}$ & 0,30 & 0,62 & 1,09 \\
\hline $0,03 \mathrm{~B} / \mathrm{V}$ & 1,20 & 1,83 & 3,77 \\
\hline $0,05 \mathrm{~B} / \mathrm{V}$ & 1,92 & 1,96 & 3,15 \\
\hline $0,08 \mathrm{~B} / \mathrm{V}$ & 1,98 & 2,99 & 4,49 \\
\hline $0,10 \mathrm{~B} / \mathrm{V}$ & 0,39 & - & 2,54 \\
\hline $0,15 \mathrm{~B} / \mathrm{V}$ & 0,46 & 0,79 & 2,52 \\
\hline $0,20 \mathrm{~B} / \mathrm{V}$ & 0,27 & 1,35 & 2,15 \\
\hline $0,25 \mathrm{~B} / \mathrm{V}$ & 1,95 & 2,52 & 3,51 \\
\hline
\end{tabular}

Как видно из полученных данных, адгезионные характеристики росли с увеличением потенциала смещения поверхности до значения $-0,08 \mathrm{~B}$. После этого значения происходило снижение прочностных характеристик покрытий. При этом необходимо отметить, что незначительное электрохимическое воздействие в 0,01 В на поверхность не способствует увеличению прочностных характеристик.

\section{Выводы}

1. Изучено влияние смещения потенциала электрода в катодную область на скорость осаждения, состав и структуру получаемых никелевых покрытий.

2. Показано, что при сдвиге потенциала в катодную область происходит соосаждение химического и электрохимического никеля, что способствует возрастанию скорости осаждения с 10 до $40 \mathrm{мкм/ч} \mathrm{(в} 4$ раза).

3. Установлено, что при смещении потенциала от стационарного значения на $-0,03$ В происходит снижение содержания фосфора в никелевом покрытии с 4,60 до 1,54\%, а при дальнейшем увеличении сдвига потенциала до -0,08 В происходит рост его содержания до 4,02 \%, вероятно, за счет прямого электрохимического восстановления гипофосфита. При этом показано, что образцы, полученные при смещениях потенциала $-0,15 ;-0,20$ и $-0,25$ В, не содержат фосфора.

4. Способ совместного нанесения химического и электрохимического никеля при некоторых смещениях потенциала поверхности в катодную область позволяет получать покрытия с меньшим содержанием фоссора, но лучшими физико-химическими характеристиками. 


\section{СПИСОК ЛИТЕРАТУРЫ}

1. Volkova I.R., Chiganova G.A., Kiseleva S.A. The Structure and Main Characteristics of Diamond-Bearing Ni-P Coatings // Protection of Metals and Physical Chemistry of Surfaces. - 2018. V. 54. - № 1. - P. 95-98.

2. Sheng G.Y. Study on Growth Mode and Properties of Electroless Nickel Plating on Aluminum Alloy // Chemical Engineering Transactions. - 2016. - V. 55. - P. 319-324.

3. Loto C.A. Electroless nickel plating - a review // Silicon. 2016. - V. 8. - № 2. - P. 177-186.

4. Mazur K., Stefańska A., Hebda M. Analysis of Chemical NickelPlating Process // Materials Science. - 2018. - V. 54. - № 3. P. 387-394.

5. Effects of Ni-P amorphous films on mechanical and corrosion properties of $\mathrm{Al} 0.3 \mathrm{CoCrFeNi}$ high-entropy alloys / Z.H. Xia, M. Zhang, Y. Zhang, Y. Zhao, P.K. Liaw, J.W/ Qiao // Intermetallics. - 2018. - V. 94. - P. 65-72.

6. Farzaneh A., Ehteshamzadeh M., Cobley A.J. Modelling of surfactants and chemistry for electroless Ni-P plating // Surface Engineering. - 2018. - V. 34. - № 6. - P. 454-461.

7. Квасова А.М., Невмятуллина Х.А., Винокуров Е.Г. Оценка функционирования процесса химического никелирования на основе SWOT-анализа // Успехи в химии и химической технологии. - 2016. - Т. 30. - № 2. - С. 14-15.

8. Pure thick nickel coating obtained by electroless plating: Surface characterization and wetting properties / V. Genova, L. Paglia, F. Marra, C. Bartuli, G. Pulci // Surface and Coatings Technology. - 2019. - V. 357. - P. 595-603.

9. Петухов И.В. Влияние концентрации компонентов раствора химического никелирования на топографию и микрорельеф Ni-P-покрытий // Электрохимия. - 2008. - Т. 44. - № 2. C. $161-172$.

10. Повышение производительности процесса химического никелирования / В.Д. Скопинцев, А.В. Моргунов, Е.Г. Винокуров, Х.А. Невмятуллина // Гальванотехника и обработка поверхности. - 2016. - Т. 24. - № 3. - С. $26-31$.

11. Electrochemically promoted electroless nickel-phosphorous plating on titanium substrate / C. Gao, L. Dai, W. Meng, Z. He,
L. Wang // Applied Surface Science. - 2017. - V. 392. P. 912-919.

12. Алексенко И.А. Химическое никелирование пластин из алюминия с применением электростимуляции поверхности // Химия и химическая технология в XXI веке: материалы XVII Международной научно-практической конференции студентов и молодых ученых имени профессора Л.П. Кулёва, посвященной 120-летию Томского политехнического университета. Томск, 17-20 мая 2016. - С. 36-37.

13. Беленький М.А., Иванов А.Ф. Электроосаждение металлических покрытий. - М.: Металлургия, 1985. - 292 с.

14. Петухов И.В. 0 механизме роста Ni-P-покрытий, получаемых методом химического осаждения // Электрохимия. - 2007. T. 43. - № 1. - C. 36-43.

15. Влияние времени осаждения на процессы формирования Ni-P покрытий / И.В. Петухов, В.В. Семенова, Н.А. Медведева, В.А. Оборин // Вестник Пермского университета. Серия: Химия. -2011 . - № 3. - С. 47-56.

16. Моисеев В.И. Методика определения атомной структуры осадков химически восстановленного никеля // Известия Академии наук СССР. Серия физическая. - 1962. - T. XXVI. № 3. - C. 378-383.

17. Горелик С.С., Добаткин С.В., Капуткина Л.М. Рекристаллизация металлов и сплавов. - М.: МИСиС, 2005. - 432 с.

18. Delaunois F., Lienard P. Heat treatments for electroless nickel-boron plating on aluminium alloys // Surface and Coatings Technology. - 2002. - V. 160. - № 2-3. - P. 239-248.

19. Ashassi-Sorkhabi H., Rafizadeh S.H. Effect of coating time and heat treatment on structures and corrosion characteristics of electroless Ni-P alloy deposits / Surface and coatings Technology. - 2004. - V. 176. - № 3. - P. 318-326.

20. Mai Q.X., Daniels R.D., Harpalani H.B. Structural changes induced by heating in electroless nickel-phosphorus alloys // Thin $\mathrm{S}_{0}$ lid Films. - 1988. - V. 166. - P. 235-247.

Поступила 12.09.2019 2.

\section{Информация об авторах}

Рогова И.А., аспирант отделения естественных наук Школы базового инженерного образования Национального исследовательского Томского политехнического университета.

Ильин А.П., доктор физико-математических наук, профессор отделения естественных наук Школы базового инженерного образования Национального исследовательского Томского политехнического университета.

Налесник О.И., кандидат технических наук, доцент научно-образовательного центра Н. М. Кижнера Инженерной школы новых производственных технологий им. Н.М. Кижнера Национального исследовательского Томского политехнического университета.

Матренин C.B., кандидат технических наук, старший преподаватель отделения материаловедения Инженерной школы новых производственных технологий Национального исследовательского Томского политехнического университета. 


\title{
INFLUENCE OF STATIONARY POTENTIAL SHIFT OF ALUMINIUM SURFACE ON PROPERTIES OF NICKEL-PHOSPHORUS COATINGS
}

Inna A. Rogova',

inna.aleksenk092@mail.ru

\author{
Aleksandr P. llyin', \\ ilyin@mail.ru \\ Oleg I. Nalesnik', \\ nalesnik@tpu.ru \\ Sergey V. Matrenin', \\ msv@mail.ru
1 National Research Tomsk Polytechnic University, 30, Lenin Avenue, Tomsk, 634050, Russia.

The relevance of the research is caused by the need to improve the already known methods of electroless coating deposition. To date, despite numerous studies and practical applications, the process of chemical nickel plating remains imperfect. The low nickel deposition rate, the high consumption of the solution components, and the complexity of the deposition do not contribute to the widespread use of chemical nickel plating. At the same time, chemically deposited coatings are significantly different from the electrochemical: they possess valuable properties. The presented technique of intensification due to the combination of nickel deposition processes by electroless and electrochemical methods is able to increase the deposition rate of the coatings, and also allows influencing their composition and mechanical properties.

The main aim of the research is to increase the rate of electroless nickel plating by application of a negative electric potential on aluminium substrate, with a thin layer of chemically predeposited nickel to reduce the time of the process.

Objects: nickel coatings on an aluminium substrate made of alloy grade D16T.

Methods: potentiodynamic, scanning electron microscopy, energy dispersive analysis, microhardness, scratch test, profilometry.

Results. Co-deposition of electroless and electrochemical nickel on an aluminium substrate was carried out with the negative electric potential shift. The dependence of phosphorus content in the coatings on the surface stationary potential shift was determined. The physicochemical characteristics of the obtained coatings were studied. It was established that a small shift of the surface stationary potential to the cathode region allows obtaining nickel-phosphorus coatings containing a smaller amount of phosphorus, but superior in properties to an electroless coating.

Key words:

Shift potential, nickel deposition rate, electroless nickel plating, aluminium, roughness, hardness, adhesion.

\section{REFERENCES}

1. Volkova I.R., Chiganova G.A., Kiseleva S.A. The Structure and Main Characteristics of Diamond-Bearing Ni-P Coatings. Protection of Metals and Physical Chemistry of Surfaces, 2018, vol. 54, no. 1, pp. 95-98.

2. Sheng G.Y. Study on Growth Mode and Properties of Electroless Nickel Plating on Aluminum Alloy. Chemical Engineering Transactions, 2016, vol. 55, pp. 319-324.

3. Loto C.A. Electroless nickel plating - a review. Silicon, 2016 , vol. 8, no. 2, pp. 177-186.

4. Mazur K., Stefańska A., Hebda M. Analysis of Chemical NickelPlating Process. Materials Science, 2018, vol. 54, no. 3, pp. 387-394.

5. Xia Z.H., Zhang M., Zhang Y., Zhao Y., Liaw P.K., Qiao J.W. Effects of Ni-P amorphous films on mechanical and corrosion properties of $\mathrm{Al} 0.3 \mathrm{CoCrFeNi}$ high-entropy alloys. Intermetallics, 2018 , vol. 94 , pp. $65-72$.

6. Farzaneh A., Ehteshamzadeh M., Cobley A.J. Modelling of surfactants and chemistry for electroless Ni-P plating. Surface Engi neering, 2018, vol. 34, no. 6, pp. 454-461.

7. Kvasova A.M., Nevmyatullina H.A., Vinokurov E.G. Evaluation of the functioning of the electroless nickel plating process based on SWOT-analysis. Advances in Chemistry and Chemical Technology, 2016, vol. 30, no. 2, pp. 14-15. In Rus.
8. Genova V., Paglia L., Marra F., Bartuli C., Pulci G. Pure thick nickel coating obtained by electroless plating: Surface characterization and wetting properties. Surface and Coatings Technology, 2019, vol. 357, pp. 595-603.

9. Petuhov I.V. Influence of the concentration of the solution components of chemical nickel plating on the topography and microrelief of Ni-P coatings. Electrochemistry, 2008, vol. 44, no. 2, pp. 161-172. In Rus.

10. Skopintsev V.D., Morgunov A.V., Vinokurov E.G., Nevmyatullina H.A. Improving the performance of the electroless nickel plating process. Electroplating and surface treatment, 2016, vol. 24, no. 3, pp. 26-31. In Rus.

11. Gao C., Dai L., Meng W., He Z., Wang L. Electrochemically promoted electroless nickel-phosphorous plating on titanium substrate. Applied Surface Science, 2017, vol. 392, pp. 912-919.

12. Aleksenko I.A. Khimicheskoe nikelirovanie plastin iz alyuminiya s primeneniem elektrostimulyatsii poverkhnosti [Electroless nickel plating of aluminium plates with electrical stimulation of surface]. Khimiya i khimicheskaya tekhnologiya $v$ XXI veke. Materialy XVII Mezhdunarodnoy nauchno-prakticheskoy konferentsii studentov i molodykh uchenykh imeni professor L.P. Kuleva, posvyashchennoy 120-letiyu Tomskogo Politekhnicheskogo universiteta [Chemistry and chemical technology in the XXI century. Proc. of the XVII International scientific-practical conference of 
studentsand young scientists named after professor L.P. Kulev, dedicated to $120^{\text {th }}$ anniversary of Tomsk Polytechnic University]. Tomsk, 17-20 May 2016. pp. 36-37.

13. Belenkiy M.A., Ivanov A.F. Elektroosazhdenie metallicheskikh pokryitiy [Electrodeposition of metallic coatings]. Moscow, Metallurgiya Publ., 1985. 292 p.

14. Petuhov I.V. On the growth mechanism of Ni-P coatings produced by electroless deposition. Electrochemistry, 2007, vol. 43, no. 1, pp. 36-43. In Rus.

15. Petuhov I.V., Semenova V.V., Medvedeva N.A., Oborin V.A. The effect of deposition time on the formation of Ni-P coatings. Bulletin of Perm University. Series: Chemistry, 2011, no. 3, pp. 47-56. In Rus.

16. Moiseev V.I. Metodika opredeleniya atomnoy struktury osadkov khimicheski vosstanovlennogo nikelya [Method for determining the atomic structure of precipitates of chemically reduced nickel]. Izvestiya Akademii nauk SSSR. Seriya fizicheskaya, 1962, vol. XXVI, no. 3, pp. 378-383.

Information about the authors

Inna A. Rogova, postgraduate student, National Research Tomsk Polytechnic University.

Aleksandr P. Ilyin, Dr. Sc., professor, National Research Tomsk Polytechnic University.

Oleg I. Nalesnik, Cand Sc., associate professor, National Research Tomsk Polytechnic University.

Sergey V. Matrenin, Cand Sc., senior lecturer, National Research Tomsk Polytechnic University.
17. Gorelik S.S., Dobatkin S.V., Kaputkina L.M. Rekristallizatsiya metallov $i$ splavov [Recrystallization of metals and alloys]. Moscow, MISIS Publ., 2005. 432 p.

18. Delaunois F., Lienard P. Heat treatments for electroless nickel-boron plating on aluminium alloys. Surface and Coatings Technology, 2002, vol. 160, no. 2-3, pp. 239-248.

19. Ashassi-Sorkhabi H., Rafizadeh S.H. Effect of coating time and heat treatment on structures and corrosion characteristics of electroless Ni-P alloy deposits. Surface and coatings Technology, 2004, vol. 176, no. 3, pp. 318-326.

20. Mai Q.X., Daniels R.D., Harpalani H.B. Structural changes induced by heating in electroless nickel-phosphorus alloys. Thin Solid Films, 1988, vol. 166, pp. 235-247.

Received: 12 September 2019. 